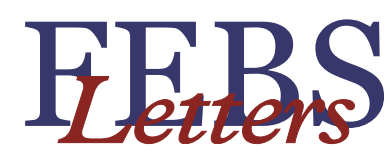

journal homepage: www.FEBSLetters.org

Review

\title{
Exploration of the spontaneous fluctuating activity of single enzyme molecules
}

\author{
Anne Schwabe a, Timo R. Maarleveld ${ }^{\mathrm{a}, \mathrm{b}, \mathrm{c}}$, Frank J. Bruggeman ${ }^{\mathrm{a}, \mathrm{d}, *}$ \\ ${ }^{a}$ Systems Bioinformatics, Amsterdam Institute for Molecules, Medicines and Systems, VU University Amsterdam, De Boelelaan 1085, 1081 HV, Amsterdam, The Netherlands \\ ${ }^{\mathrm{b}}$ Life Sciences, Centre for Mathematics and Computer Science (CWI), Science Park 123, 1098 XG, Amsterdam, The Netherlands \\ ${ }^{\mathrm{c}}$ BioSolar Cells, P.O. Box 98, $6700 \mathrm{AB}$, Wageningen, The Netherlands \\ ${ }^{\mathrm{d}}$ Kluyver Centre for Genomics of Industrial Fermentation/NCSB, P.O. Box 5057, 2600 GA, Delft, The Netherlands
}

\section{A R T I C L E I N F O}

\section{Article history:}

Received 7 June 2013

Revised 1 July 2013

Accepted 2 July 2013

Available online 12 July 2013

Edited by Athel Cornish-Bowden

\section{Keywords:}

Enzyme kinetic

Michaelis-Menten

Stochastic process

Mathematical Modeling

Single-molecule phenomenon

Biophysics

\begin{abstract}
A B S T R A C T
Single enzyme molecules display inevitable, stochastic fluctuations in their catalytic activity. In metabolism, for instance, the stochastic activity of individual enzymes is averaged out due to their high copy numbers per single cell. However, many processes inside cells rely on single enzyme activity, such as transcription, replication, translation, and histone modifications. Here we introduce the main theoretical concepts of stochastic single-enzyme activity starting from the Michaelis-Menten enzyme mechanism. Next, we discuss stochasticity of multi-substrate enzymes, of enzymes and receptors with multiple conformational states and finally, how fluctuations in receptor activity arise from fluctuations in signal concentration. This paper aims to introduce the exciting field of single-molecule enzyme kinetics and stochasticity to a wider audience of biochemists and systems biologists.
\end{abstract}

๑) 2013 Published by Elsevier B.V. on behalf of the Federation of European Biochemical Societies.

\section{Introduction}

Essentially all cellular reactions are catalyzed by enzymes, regardless of whether metabolism, signal transduction, or gene expression is considered. In metabolism, enzymes interconvert metabolites to generate the energy and precursors for macromolecular synthesis. As passive or active transporters, enzymes transport molecules in and out of the cell. Other enzymes covalently modify signaling proteins, metabolic enzymes, and nucleosomes, or transport macromolecules across the cell by free energy-driven translocation along the cytoskeleton. Thus, enzymes can operate as catalysts, either coupled to free energy transduction or not, or as molecular motors. In the latter case free energy dissipation is coupled to, for instance, directed motion of enzymes along actin or to the bacterial flagella in chemotaxis. When environmental conditions change, organisms adjust enzyme concentrations to rewire their molecular networks to better meet current demands. Natural selection acts on beneficial, genetic mutations that alter the

\footnotetext{
* Corresponding author at: Systems Bioinformatics, VU University, De Boelelaan 1085, 1081 HV, Amsterdam, The Netherlands. Fax: +31 205987229.

E-mail addresses: a.schwabe@vu.nl (A. Schwabe), t.r.maarleveld@cwi.nl (T.R Maarleveld), f.j.bruggeman@vu.nl (F.J. Bruggeman).
}

concentrations or kinetic properties of enzymes. In other words, enzymes lie at the basis of cell functioning and are central to any quantitative understanding in cell biology. Moreover, molecular systems biology studies require knowledge of enzyme properties to be able to assess how the concerted activities of enzymes, organized in networks, give rise to cell function.

The quantitative understanding of enzyme kinetics was pioneered by Michaelis and Menten in 1913 [1], followed by Briggs and Haldane in 1925 [2]. About 50 years later, Cleland unified most of this work in a comprehensive theory of monomeric enzyme kinetics in a series of seminal papers [3-5]. The work about feedback-regulation of metabolic enzymes [6,7] initiated studies on the kinetics of oligomeric enzymes, which were later followed by many studies on hemoglobin (reviewed in [8]). Oligomeric enzymes are composed out of several, interacting subunits that can display cooperativity and may be under allosteric control [9]. This lead to the development of allosteric and cooperative enzyme kinetics: the concerted symmetry model of Monod, Wyman, and Changeux [10], the sequential model by Koshland, Nemethy, and Filmer [11], and more recently, the reversible Hill equation by Hofmeyr and Cornish-Bowden [12]. All these theories of enzymology are macroscopic theories of enzyme kinetics, considering the average properties of ensembles of enzymes. 
Macroscopic theories in enzymology about monomeric and multimeric enzyme kinetics do not refer to the inherently stochastic aspects of the activity of single enzyme molecules. Enzyme ensembles concern thousands to millions of proteins that each function independently. Much can be learned about enzymes from those studies. For example, in metabolism the simultaneous activity of a huge number of enzymes matters most often. Consequently, the ensemble perspective gives the relevant picture. However, from the study of single enzyme molecules valuable additional insight can be gained about enzyme mechanisms $[13,14]$. Moreover, in many cellular functions it is the activity of single enzymes that matters. Examples are protein transport along the cytoskeleton or mRNA synthesis via transcription, which are inherently single-enzyme activities. In those latter examples, spontaneous, stochastic fluctuations in the activity of a single enzyme can have profound consequences that can be propagated to the phenotypic properties of a cell, on which natural selection acts. Thus in those cases the study of single enzyme molecules is required to understand fundamental aspects of cell biology.

The experimental study of single enzyme copies was pioneered by B. Rotman in 1961 [15], but gained real momentum only three decades later with the advance of single-molecule fluorescence microscopy techniques (reviewed in [14]). Here we limit ourselves to the theoretical aspects of the activity of single enzyme molecules. We use several illustrative examples of the activity of single enzyme molecules to introduce the reader to the main stochastic models (Supplemental material) and concepts of this exciting field in enzymology.

\section{Results}

\subsection{Fluctuations in the activity of a Michaelis-Menten enzyme}

In this section we derive some of the microscopic properties of enzyme kinetics, which concern the stochastic activity of the single enzyme molecules, and relate those to classical enzyme kinetic parameters (i.e. $K_{M}$ and $V_{M A X}$ ). In particular, we discuss the distribution of times between subsequent product formations catalyzed by a single enzyme, the turnover time distribution, and how this relates to the catalysis rate of the enzyme. In the Supplemental material we present two methods how this can be done for arbitrarily complex enzyme mechanisms. When the Michaelis-Menten (MM) mechanism is considered, the mean turnover time turns out to give rise to the well-known MM rate equation.

The simplest model that gives rise to the MM enzyme mechanism considers one enzyme $(E)$, one substrate $(S)$, one enzyme-substrate complex $(E S)$, and one product $(P)$. The enzyme-substrate complex can either dissociate into the enzyme and substrate or catalysis can occur giving rise to product and the original enzyme (Fig. 1A).

Deterministic models of enzyme activity fail to capture the discreteness and stochasticity effects that occur when enzyme molecules are present at low copy numbers. If we assume that the cell is a well-stirred compartment, we can ignore molecular positions and diffusion. Then, we can use the chemical master equation (CME) [16] to describe the stochastic activity of a single enzyme (reviewed in [17]). The CME determines the probability that the system is in a specific state at a given (future) time. A single state is a particular combination of the number of $E, E S, S$ and $P$ molecules per cell. Note that from the stochastic perspective the state is a vector of integers (the (copy) number of a specific molecule per cell) and not of real numbers as one would have in a deterministic, macroscopic description when concentrations are considered. The general description of the CME is given in Eq. (S-6) and describes the rate of change in the probability mass to observe the system in a particular state. It is a balance equation for the probability mass of the states. A more specific description of the CME is given by: $\frac{d P\left(\boldsymbol{x}, t \mid \boldsymbol{x}_{\boldsymbol{0}}, t_{0}\right)}{d t}=\sum_{r \in R} a_{r}\left(\boldsymbol{x}-\boldsymbol{v}_{r}\right) \cdot P\left(\boldsymbol{x}-\boldsymbol{v}_{r}, t \mid \boldsymbol{x}_{\mathbf{0}}, t_{0}\right)-\sum_{r \in R} a_{r}(\boldsymbol{x}) \cdot P\left(\boldsymbol{x}, t \mid \boldsymbol{x}_{\mathbf{0}}, t_{0}\right)$

Here, $\boldsymbol{x}$ is the state vector which contains the number of molecules (denoted later by $N$ ) of each species in time, $P\left(\boldsymbol{x}, t \mid \boldsymbol{x}_{\mathbf{0}}, t_{0}\right)$ is the probability to observe the system in state $\boldsymbol{x}$ at time $t$ given the initial state $\boldsymbol{x}_{\mathbf{0}}$ at time $t_{0}, \boldsymbol{v}_{\boldsymbol{r}}$ is the state-change vector of reaction $r$ (vector with stoichiometric coefficients) and $a_{r}(\boldsymbol{x})$ is the propensity function of reaction $r$, i.e., the probability per unit time that reaction $r$ fires given that the system is currently in state $\boldsymbol{x}$.

For the simple model shown in Fig. 1A, the propensity functions are given by (very similar to mass-action kinetics),

$$
\begin{aligned}
& a_{1}(\boldsymbol{x})=k_{1}^{+} \cdot N_{E} \cdot N_{S} \\
& a_{2}(\boldsymbol{x})=k_{1}^{-} \cdot N_{E S} \\
& a_{3}(\boldsymbol{x})=k_{c} \cdot N_{E S}
\end{aligned}
$$

where $N_{E}, N_{S}$, and $N_{E S}$ are the copy numbers - number of molecules - of $E, S$, and $E S$, respectively. Note that rate constants of unimolecular reactions are independent of the system volume $(V)$, whereas rate constants of bimolecular reactions (e.g. $k_{1}^{+}$) are inversely proportional to $V$. This is because the search time for two reactant molecules depends on volume [18].

If we assume that the number of substrate molecules, $N_{S}$, is held constant the state-change vectors for the simple enzyme kinetics model are given by,

$$
\begin{aligned}
& \boldsymbol{v}_{\mathbf{1}}=(-1,1,0) \\
& \boldsymbol{v}_{\mathbf{2}}=(1,-1,0) \\
& \boldsymbol{v}_{\mathbf{3}}=(1,-1,1)
\end{aligned}
$$

of which the entries correspond to the stoichiometric coefficients of the molecules in the same order as they occur in the state vector $\boldsymbol{x}$ :

$\boldsymbol{x}=\left(N_{E}, N_{E S}, N_{P}\right)$

Thus, reaction (2) produces one molecule $E$ and consumes one molecule ES ( $S$ is considered fixed and therefore not specified).

We consider only a single enzyme copy, which implies that $N_{E S}$ is 0 if $N_{E}$ is 1 and vice versa (Fig. 1B and 1C). Production events of $P$ will occur at irregular intervals when a single enzyme is modeled with the CME. An example is shown in Fig. 1D where three different trajectories of a stochastic simulation are shown. Each simulation started from the same initial conditions and with the same kinetic parameters. These trajectories fluctuate around the analytical solution for a large ensemble of the same enzyme molecules. This ensemble is described by the set of ordinary differential equations that characterize the deterministic, macroscopic dynamics of an ensemble of independent enzyme molecules.

We are interested in the time to make one product molecule, i.e., to increase $N_{P}$ by 1 . Directly after the previous product molecule has been synthesized the enzyme is in the unbound state. Therefore, we consider an initial condition with $N_{E}(0)=1$, $N_{E S}(0)=0$ and use the CME to determine how the probability $P\left(N_{E}, N_{E S}, N_{P}, t\right)$ changes over time from this initial condition. Note that the number of molecules of $P$ does not matter in this case, as it does not influence any of the elementary enzyme rates.

Substituting Eqs. (2)-(4) into Eq. (1) gives the CME for our simple enzyme kinetics model:

$$
\begin{aligned}
\frac{d P\left(1,0, N_{P}, t \mid x_{0}, t_{0}\right)}{d t}= & k_{1}^{-} \cdot P\left(0,1, N_{P}, t \mid x_{0}, t_{0}\right)+k_{c} \cdot P\left(0,1, N_{P}\right. \\
- & \left.1, t \mid x_{0}, t_{0}\right)-k_{1}^{+} \cdot N_{S} \cdot P\left(1,0, N_{P}, t \mid x_{0}, t_{0}\right) \\
& \times \frac{d P\left(0,1, N_{P}, t \mid x_{0}, t_{0}\right)}{d t} \\
= & k_{1}^{+} \cdot N_{S} \cdot P\left(1,0, N_{P}, t \mid x_{0}, t_{0}\right)-\left(k_{1}^{-}+k_{c}\right) \\
& \cdot P\left(0,1, N_{P}, t \mid x_{0}, t_{0}\right)
\end{aligned}
$$


A
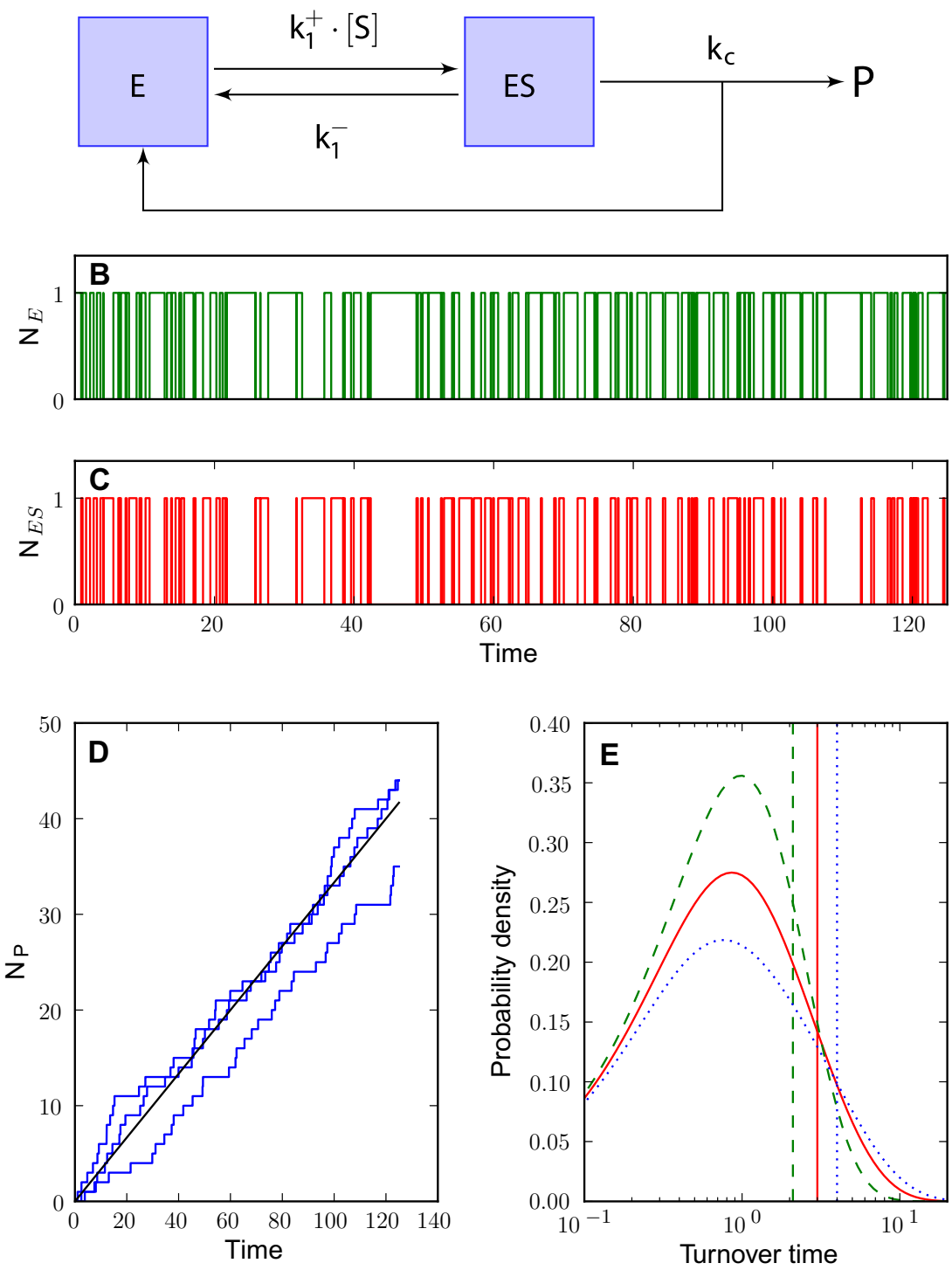

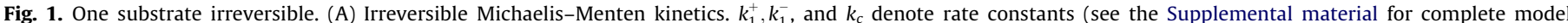

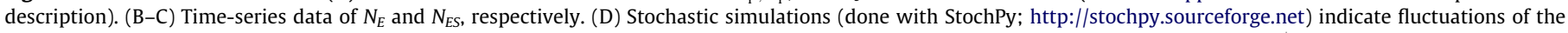

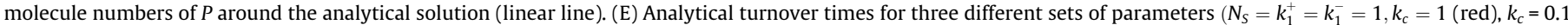
(green, dashed), and $k_{c}=2$ (blue, dotted). Vertical lines illustrate the mean turnover times corresponding to the MM equation.

Since we are considering a single enzyme molecule we have the constraint $N_{E}+N_{E S}=1$ whereas the values of $N_{P}$ can take any positive discrete value. With initial conditions as defined above and $t_{0}=0$, the probability that a product is formed after time $t$ equals the product of the catalytic rate $\left(k_{c}\right)$ and the probability that the enzyme is in the substrate bound state,

$f(t)=k_{c} \cdot P\left(0,1, N_{P}, t \mid N_{E}(0)=1, N_{E S}(0)=0\right)$

$$
=\frac{a\left(e^{(-c+b) t / 2}-e^{(-c-b) t / 2}\right)}{b}
$$

with $\quad a=k_{1}^{+} k_{c} N_{S}, b=\sqrt{-4 k_{1}^{+} k_{c} N_{S}+\left(k_{1}^{-}+k_{c}+k_{1}^{+} N_{S}\right)^{2}}$, and $c=k_{1}^{-}+k_{c}+k_{1}^{+} N_{s}$. This distribution is illustrated in Fig. 1E for three different sets of parameters where the vertical lines illustrate the mean turnover time, $\langle t\rangle$. To obtain Eq. (6) we solved the CME given in Eq. (5) at time equals 0 . Then for $N_{P}=0$, we have $P\left(0,1, N_{p}-1, t \mid x_{0}, t_{0}\right)=0$ and the initial conditions for the system of equations in (5) becomes $P\left(1,0, N_{p}, t \mid x_{0}, t_{0}\right)=1$ and $P\left(0,1, N_{p}, t \mid x_{0}, t_{0}\right)=$ 0 . The resulting two linear differential equations can be solved analytically to give rise to Eq. (6).
Subsequently, the mean turnover time can be calculated from (6):

$\langle t\rangle=\int_{0}^{\infty} t f(t) d t=\frac{k_{1}^{-}+k_{c}+k_{1}^{+} N_{S}}{k_{c} k_{1}^{+} N_{S}}=\frac{\frac{k_{1}^{-}+k_{c}}{k_{1}^{+}}+N_{S}}{k_{c} N_{S}} \stackrel{\text { def }}{=} \frac{K_{m}+N_{S}}{V_{\max } N_{S}}$

Here we identify the MM constant, $K_{M}=\frac{k_{1}^{-}+k_{c}}{k_{1}^{+}}$, and, the maximal enzyme rate, $V_{\text {max }}=k_{c}\left(N_{E}+N_{E S}\right)=k_{c}$. Hence, the mean turnover time $\langle t\rangle$ equals the inverse of the catalysis rate of the enzyme as described by the MM rate equation. In general, the mean turnover time for any enzyme mechanism equals the inverse of the catalysis rate as derived from the quasi-steady state assumption. Thus, the deterministic, macroscopic model of classical enzyme kinetics considers the mean activity of an ensemble of independent enzyme molecules. Eq. (6) describes the stochasticity in the turnover times between consecutive product molecules that arise due to the fluctuating catalytic activity of a single enzyme molecule.

Following the reasoning of Qian [19] we can decompose $\langle t\rangle$ into a sum of dwell times of each state multiplied with the average 
number of times, $n$, that the $E$ and $E S$ states are visited before a product molecule is formed:

$\langle t\rangle=(\underbrace{\frac{1}{k_{1}^{+} N_{S}}}_{\left\langle t_{E}\right\rangle}+\underbrace{\frac{1}{k_{1}^{-}+k_{c}}}_{\left\langle t_{E S}\right\rangle}) \underbrace{\frac{k_{c}+k_{1}^{-}}{k_{c}}}_{n}$

Here $\left\langle t_{E}\right\rangle$ and $\left\langle t_{E S}\right\rangle$, respectively, equal the life time of the $E$ and the $E S$ state and the factor $n$ expresses the number of enzyme cycles $E S \rightarrow E+S \rightarrow E S$ up to a productive event $E S \rightarrow E+P$.

To characterize the stochasticity in the production process, we determine the noise of the turnover time distribution. The noise is given by the variance in the turnover time, $\left\langle\delta^{2} t\right\rangle$, divided by the squared mean turnover time:

$\frac{\left\langle\delta^{2} t\right\rangle}{\langle t\rangle^{2}}=\frac{\left\langle t^{2}\right\rangle-\langle t\rangle^{2}}{\langle t\rangle^{2}}=\left(1+\frac{2 k^{+} k_{c} N_{S}}{\left(k_{1}^{-}+k_{c}\right)^{2}+2 k_{1}^{-} k_{1}^{+} N_{S}+\left(k_{1}^{+}\right)^{2} N_{S}^{2}}\right)^{-1}$

In the limit of either very low or very high substrate molecule numbers, the turnover time distribution approaches an exponential turnover time distribution. Accordingly, the turnover noise approaches 1 . For instance, when $N_{S}$ becomes high, the mean turnover becomes $1 / k_{c}$; the catalytic step becomes rate limiting. For intermediate substrate concentrations the noise is lower than 1 . In other words, the two-step nature of the process (binding plus catalysis) reduces the noise below that of an exponential distribution. We note that exponential distribution is often considered a "noisy" distribution, because its mean and standard deviation are equal.

\subsection{Universality of the Michaelis-Menten equation from a single- molecule perspective}

In the previous section we have identified the classical MM equation as the inverse of the mean turnover time of single enzyme molecule, following the MM mechanism (Fig. 1). Most enzymatic reactions, however, do not follow this simple scheme. Firstly, bi-substrate reactions account for roughly $60 \%$ of known enzymatic reactions. Secondly, in many cases reactions cannot be considered irreversible. Thirdly, many enzymatic reactions involve multiple intermediate states. Nonetheless, the MM equation has been applied successfully to many such enzymes. In this section, we take a look at some of those mechanisms, again from a single-molecule perspective and show which types of mechanisms do or do not lead to the well-known hyperbolic substrate dependence.

Bi-substrate mechanisms are usually classified according to a scheme developed by Cleland [3], for instance, as sequential (either ordered or random) or as ping pong. We will start by deriving and comparing the mean turnover times, $\langle t\rangle$, as a function of both substrates for the two sequential mechanisms depicted in Fig. 2A. Using Eq. (S-4) one obtains:

$$
\begin{aligned}
& \left\langle t_{\text {ordered }}\right\rangle=a_{0}+\frac{a_{1}}{N_{S_{1}}}+\frac{a_{2}}{N_{S_{2}}}+\frac{a_{3}}{N_{S_{1}} N_{S_{2}}} \\
& \left\langle t_{\text {random }}\right\rangle=\frac{a_{0}+a_{1} N_{S_{1}}+a_{2} N_{S_{1}}^{2}}{a_{3} N_{S_{1}}+a_{4} N_{S_{1}}^{2}}
\end{aligned}
$$

where for the random mechanism the number of molecules of the second substrate, $N_{S_{2}}$, is considered constant (an analogous expression is obtained when $N_{S_{1}}$ is constant and substrate two is varied). The $a_{i}$ are coefficients that depend only on the rate constants and the concentration of the fixed substrate. The ordered sequential mechanism obeys the MM equation for both substrates. More specifically, when one substrate concentration is kept fixed, the mean turnover time depends linearly on the inverse of the other substrate
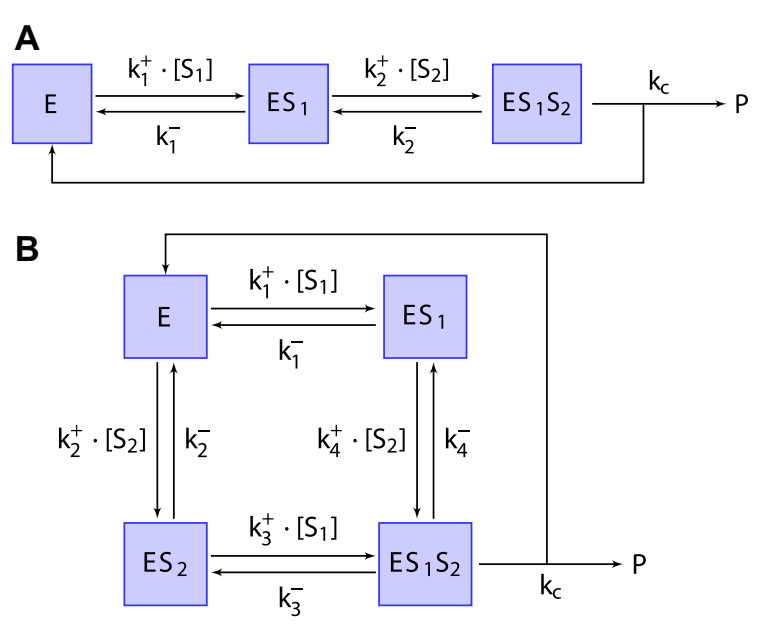

Fig. 2. Two substrates. Models where two substrates bind in either a sequential manner (A) or a random manner (B). See the Supplemental material for complete model description.

concentration. This is, however, not true for the random sequential mechanism, only in the limit of saturating concentrations of the fixed substrate. Where does this difference come from?

Again, we can decompose the mean turnover time into the mean duration spent in each state multiplied by the average number of times that this state is visited before a product molecule is formed. For the sequential mechanism the dwell times in the states are $\left\langle t_{E}\right\rangle=\frac{1}{k_{1}^{+} N_{S_{1}}},\left\langle t_{E S 1}\right\rangle=\frac{1}{k_{1}^{-}+k_{2}^{+} N_{S_{2}}}$, and $\left\langle t_{E S 1 S 2}\right\rangle=\frac{1}{k_{2}+k_{c}}$. Any linear combination of those dwell times - as long as the coefficients do not depend on $N_{S_{1}}$ themselves - will yield a linear relationship between $\langle t\rangle$ and $1 / N_{S_{1}}$. For $N_{S_{2}}$ the derivation is a bit more involved. The average number of times that the enzyme goes through state ES1S2 before a product is formed is given by

$\left(\frac{k_{1}^{-}+k_{2}^{+} N_{S_{2}}}{k_{2}^{+} N_{S_{2}}}\right)\left(\frac{k_{c}+k_{2}^{-}}{k_{c}}\right)$

Here, the first term is the inverse of the probability that when the enzyme is in the ES1 state it will continue to the ES1S2 state. The second term is the inverse of the probability that product formation occurs when the system is in the ES1S2 state. Multiplying Eq. (12) with the average dwell time in the ES1 state results in the $1 / N_{S_{2}}$ relationship.

For the random mechanism the dwell times equal $\left\langle t_{E}\right\rangle=\frac{1}{k_{1}^{+} N_{S_{1}}+k_{2}^{+} N_{S_{2}}},\left\langle t_{E S 1}\right\rangle=\frac{1}{k_{1}^{-}+k_{4}^{+} N_{S_{2}}},\left\langle t_{E S 2}\right\rangle=\frac{1}{k_{2}^{-}+k_{3}^{+} N_{S_{1}}}, \quad$ and $\left\langle t_{E S 1 S 2}\right\rangle=\frac{1}{k_{3}^{-}+k_{4}^{-}+k_{c}}$. A linear combination of these dwell times does not result in a linear dependence on the inverse of the substrate concentration. The reason is that binding of a substrate to two (or more) possible states - in the example $S 1$ can bind to the free enzyme but also to the ES2 complex - leads to multiple dwell time terms of the form $\frac{1}{a+k N_{s}}$. In general, the average number of times that the state is visited does not lead to cancellation of the denominator. In a similar way as the bi-substrate sequential ordered mechanisms, the mean turnover times for models with multiple intermediate states can be derived. Such models have been used to describe the enzymatic action of ribosomes [20] and molecular motors [21]. If all states are visited in a sequential order, the MM equation holds for the inverse of the mean turnover times of those enzyme mechanisms.

\subsection{Stochastic switching between enzyme conformations}

Single-molecule studies of a number of single-substrate enzymes indicate that the average time between turnovers obeys 
the MM kinetics. However, these measurements also show that the turnover time distribution and the correlations between subsequent turnover times do not fit the equations for the simple model discussed in Section 2.1 [13,22-25]. Instead, the results are better explained by a model where the enzyme can switch between multiple conformations that have different catalytic activities and/or substrate affinities. This phenomenon is called dynamic disorder. (Note that inhibitors can cause the same behavior. In this case the enzyme switches between inhibitor bound and unbound states [26].)

Here, we illustrate dynamic disorder for a simple model with two different conformations shown in Fig. 3A. Although most experimental data could only be fitted to models with more than 10 states, a two state model does give insight into how the turnover time distribution and the turnover time correlations change in comparison to the one state model. We consider two conformations that have the same substrate association and dissociation rate constants $\left(k^{+}\right.$and $\left.k^{-}\right)$but that differ in the catalytic rate constant, $k_{c}$. While the turnover time distribution for the model without conformational changes is given by a single exponential rise and a single exponential decay (Eq. (6)), multiple conformations lead to multiple exponentials in the decay of the distribution. The two timescales become apparent when the switching between the conformations is slow in comparison with the catalytic rates. This results in bimodal turnover time distributions as is illustrated in Fig. 3B.

For a two conformation model the average turnover time can be expressed as

$\langle t\rangle=\frac{b_{0}+b_{1} N_{S}+b_{2} N_{S}^{2}}{b_{3} N_{S}+b_{4} N_{S}^{2}}$

where the coefficients $b_{0}$ to $b_{4}$ are functions of the kinetic parameters. Therefore, in general this model does not follow the MM kinetics. However, there exist a number of conditions where $\langle t\rangle$ is approximately linearly dependent on $1 / N_{S}$ [27-31]. The most biologically relevant condition is where the conversions between conformations are slow in comparison to the catalytic rate (quasi-steady state condition). Under that condition, and if the only difference between conformations is in the catalytic rate constant, the inverse of the average turnover time equals Eq. (14) [13],

$\frac{1}{\langle t\rangle}=\frac{V_{\max }[S]}{[S]+K_{m}}$

$V_{\max }=\left(\sum_{i} \frac{w_{i}}{k_{\text {cat }, i}}\right)^{-1}$

$K_{m}=\frac{V_{\max }+k_{-}}{k_{+}}$

Here, the summation runs over all conformations and the $w_{i}$ denote weights corresponding to the probability of the conformations. This illustrates that although the average turnover time has the same substrate dependency as in the classical MM equation, its microscopic interpretation is different.

In the experimental data, the multi-exponential decay of the turnover time distribution is observed only at sufficiently high substrate concentrations. At low concentrations, the binding of substrate is rate limiting and the shape of the turnover time distribution resembles that given in Eq. (6). Besides the mean turnover time as a function of substrate concentration, also its noise $\left\langle\delta^{2} t\right\rangle /\langle t\rangle^{2}$ is instructive to investigate. This value is sometimes called the randomness parameter [32]. It contains information about the type of dynamic disorder.

Without dynamic disorder, the noise in the turnover time distribution for very low and very high substrate concentrations approaches 1 . Under those conditions, the shape of the turnover
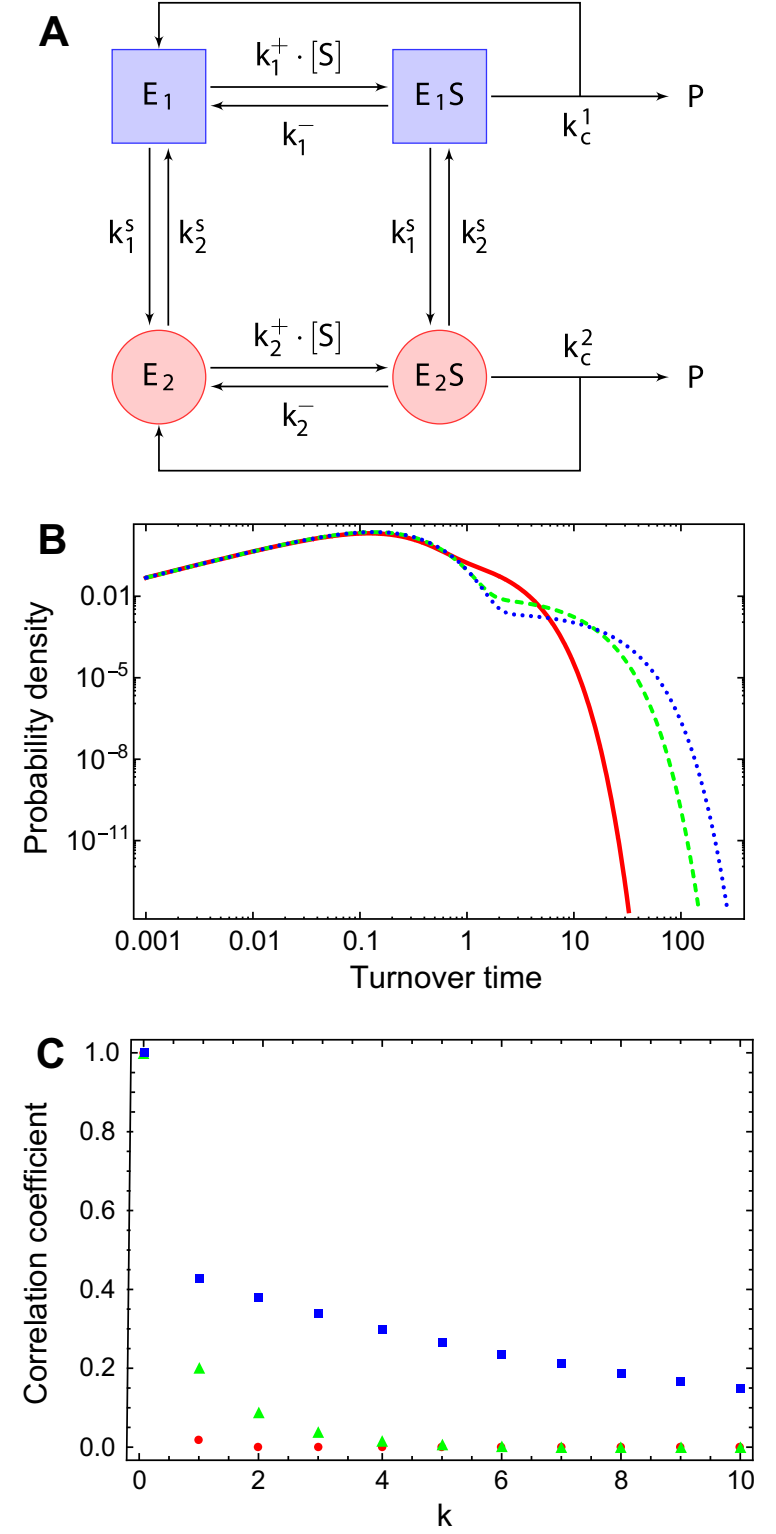

Fig. 3. Stochastic switching. (A) Enzyme switching between two conformations (see Supplemental material for complete model description). (B) Inter-event times probability density function. (C) turnover time $(\operatorname{lag}(\mathrm{k}))$ autocorrelation coefficients. $k_{1}^{+}=k_{1}^{-}=k_{1}^{+}=k_{1}^{-}=1,[S]=5, k_{c}^{1}=100, k_{c}^{2}=10, k_{1}^{s}=k_{2}^{s}=x$, with $x=1$ (red, solid, circle), $x=0.1$ (green, dashed, triangle), $x=0.01$ (blue, dotted, square).

time distribution is determined by a single rate limiting step (either substrate binding or catalysis). This gives rise to an exponential turnover time distribution. For intermediate substrate concentrations there are two reaction steps that occur on comparable timescales. Consequently, the noise can become lower than 1 . In contrast, the noise in turnover times can become larger than 1 with dynamic disorder in the catalytic rates. In the limit of slow conversion between conformations, the turnover time distribution becomes a weighted average of the turnover distributions for each conformation. This results into a higher noise than in each individual distribution. Dynamic disorder in the dissociation rate constant, however, does not increase the noise above one [30].

In addition to the turnover time distribution, a time trace of turnovers for a single enzyme contains information about correlations between subsequent turnover times. These correlations can be compared to model predictions. There are two different measures for the autocorrelation of such time traces. The first of these 
measures is the turnover time correlation (sometimes called lag $(\mathrm{k})$ autocorrelation). This is the correlation between one turnover and its $k$ th successor. The second of these measures is signal correlation. Signal correlations are defined as the autocorrelation of the signal function. This is a sum of Dirac delta functions of the times, $t_{i}$, at which product formation occurred, $s(t)=\sum_{i} \delta\left(t-t_{i}\right)$.

The application of both types of correlation functions to singlemolecule MM kinetics is analyzed in detail elsewhere [33]. The advantage of the latter is that it reflects the true timescale of the correlations. Nonetheless, for many experimental observations the signal function is not zero between turnover times. In addition, it does not have the Dirac delta as shape for a signal (usually the signal is detected over finite time and its intensity varies between events). This can lead to aberrations in comparison with theoretical predictions from idealized signal functions, especially around $t=0$.

Using Eq. (S-5) we can calculate the turnover time correlation from the rate parameters. This type of correlation distinguishes between models with and without conformational switches. For models with a single catalytically active conformation, the turnover time correlation is equal to zero for $k>0$ and it becomes positive for models with multiple conformations. This is not true for the signal autocorrelation function which can have positive values for $t>0$ also with a single conformation enzyme. In Fig. 3C we illustrate that the slower the switching between conformations the slower is the decrease in correlation with increasing $k$. Note that fluctuations in the substrate level can also introduce correlations in both types of correlation functions.

\subsection{G-protein coupled receptors}

Many types of signaling receptors (or transcription factors) could be considered as a special case of the model with multiple conformations discussed in the previous section. These receptors switch between two (main) states of which only one is active for signaling. Typically, switches occur because of a conformational change bringing about a change in conformation of an intracellular domain to affect intracellular signaling. Binding of the receptor ligand - the signal - then shifts the equilibrium between the two receptor conformations. Fig. $4 \mathrm{~A}$ shows a simple model of such a two-state receptor where binding of ligand $(L)$ induces a shift from state $R_{1}$ to $R_{2}$. An example of such receptors are G-protein coupled receptors; in contrast, receptor tyrosine kinases (RTKs) require ATP hydrolysis for conformation changes.

In the model we consider, only the $R_{2}$ state can bind to the intracellular substrate $\left(S\right.$; e.g. $\left.G^{\mathrm{GDP}}\right)$ and convert it to a product $\left(P\right.$; e.g. $\left.G^{\mathrm{GTP}}\right)$. This product can be thought of as a second messenger; a small molecule that transmits the information from the membrane receptor to downstream intracellular signaling processes. With only one of the two states being active, the production of second messengers from a single receptor molecule can occur in bursts. A production period is then followed by a period of inactivity while the receptor exists in $R_{1}$ conformation (Fig. $4 \mathrm{~B}$ ).

The product $(P)$ time traces (Fig. 4B) are comparable to those observed in transcriptional bursting [34]. The turnover time distribution (Fig. 4C) shows the two timescales characteristic for bursting systems [34]. The distributions of times spent in the active and inactive states can be calculated in an analogous manner as the turnover time distribution (Supplemental material Eq. (S-1)).

The burst size distribution can also be derived analytically [35]. If switching between the ligand bound and the free receptor state is faster than switching between active and inactive states, the durations of active and inactive periods approach exponential distributions and the burst size becomes geometrically distributed. At the other extreme, if ligand association and dissociation is slow in comparison to switching between active and inactive states, those durations follow distributions with bi-exponential decay.
If signaling by a single cell would occur via a single receptor molecule, the bursting activity of second messenger production would limit information transmission. Unless, of course, the life time of the second messenger is long enough such that fluctuations in its production rate due to bursting are effectively averaged out. However, this picture changes when multiple receptors are independently signaling at the level of a single cell. Since, their activity periods are then out of synchrony, bursting is reduced very effectively. This is illustrated in Fig. 4B and C where we depict the turnover time distributions and the time traces of product formation for one, three, and ten independent receptors. The noise in turnover time decreases from 18.2 (for the single receptor) to 1.1 (for the ensemble of ten receptors). A bacterium with ten receptors per cell would therefore not experience bursting in intracellular signaling processes. Eukaryotes tend to have thousands of receptors per cell.

For any type of turnover time distribution, the superposition of several processes with this distribution will lead to an aggregate turnover time distribution that converges to an exponential distribution when the number of super imposed processes goes to infinity [36]. Interestingly, ten receptors already come very close to generating an exponential turnover time distribution. Therefore, even for a small organism like Escherichia coli the noise in the turnover time distribution of a single receptor or transcription factor can be expected not to be relevant for signaling or transcription; unless their copy numbers are between 1 and 5 per cell, which is the case for some transcription factors in E. coli. There exists an additional source of signaling noise that derives from stochastic fluctuations in the ligand concentration that regulates the receptor. This we will discuss in the following section.

\subsection{Fluctuating ligand concentrations in signaling can cause signaling errors}

Cells derive information about the concentration of an extracellular ligand from the fractional occupancy of receptors (occupancy probability; fraction of time that the receptor is bound to a signal molecule). Starting from the active receptor the signal is transmitted into the cell. Each step in the signal transmission has its own noise causing little errors in signal transmission that accumulate. What sets the fundamental precision limits with which a cell can sense the concentration of an extracellular ligand? It turns out that those limits are set by at least two factors. The first factor is the speed of diffusion with which ligand molecules arrive at the cellular membrane. The second factor is the time period over which the receptor occupancy is averaged before an intracellular signaling event is initiated. Berg and Purcell [37] and later (using fewer assumptions) Bialek and Setayeshgar [38] derived these limits. Here we will shortly review them as an example of noise at the single enzyme level that is not due to the enzyme mechanism but derives from the noise in an allosteric regulator.

For a receptor with radius $s$ the average number of molecules, $n$, in the receptor's detection volume is proportional to $c \cdot s^{3}$, with $c$ as the extracellular ligand concentration. The number of molecules in this volume follows a Poisson distribution (zero-order influx and first-order efflux from the volume). Thus, the variance in this molecule number will equal the mean molecule number. As a result, the standard deviation becomes, $\delta n=\left(c \cdot s^{3}\right)^{1 / 2}$. This is the "error" in the ligand copy number that a single receptor molecule experiences.

Measurement of the same molecule that was bound earlier to the same receptor does not improve the estimate of extracellular concentration, as it is not an independent measurement. Re-equilibration of the ligand molecules in the volume $s^{3}$ with the bulk solution is required for an independent measurement. A time average over the receptor occupancy does, therefore, reduce the 
A

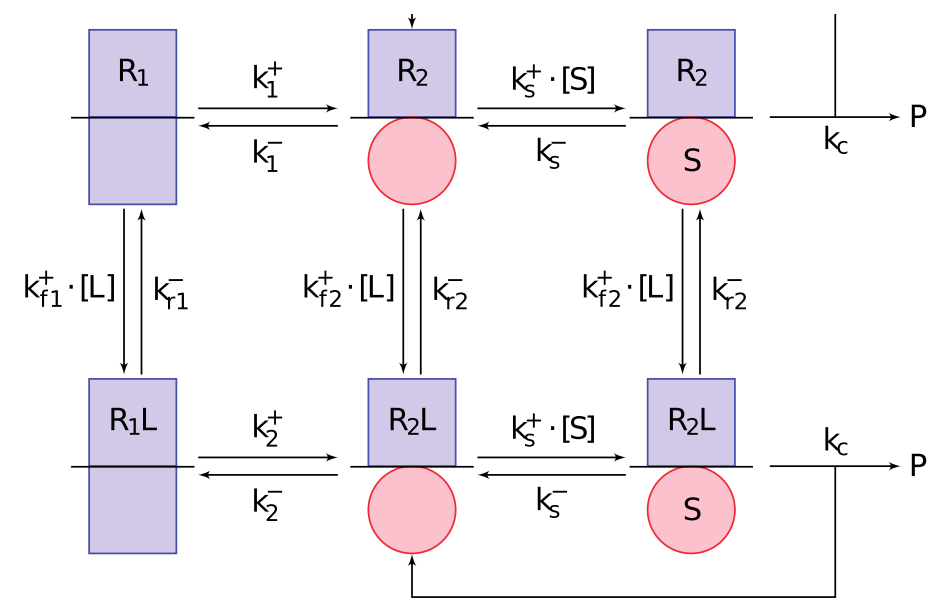

B

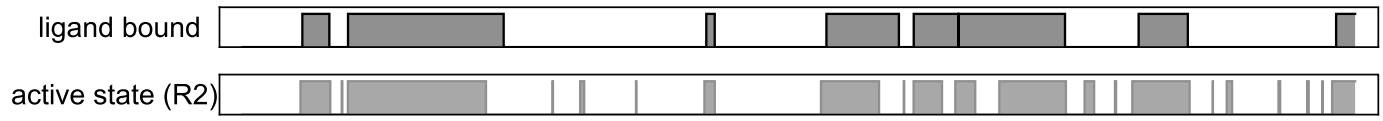
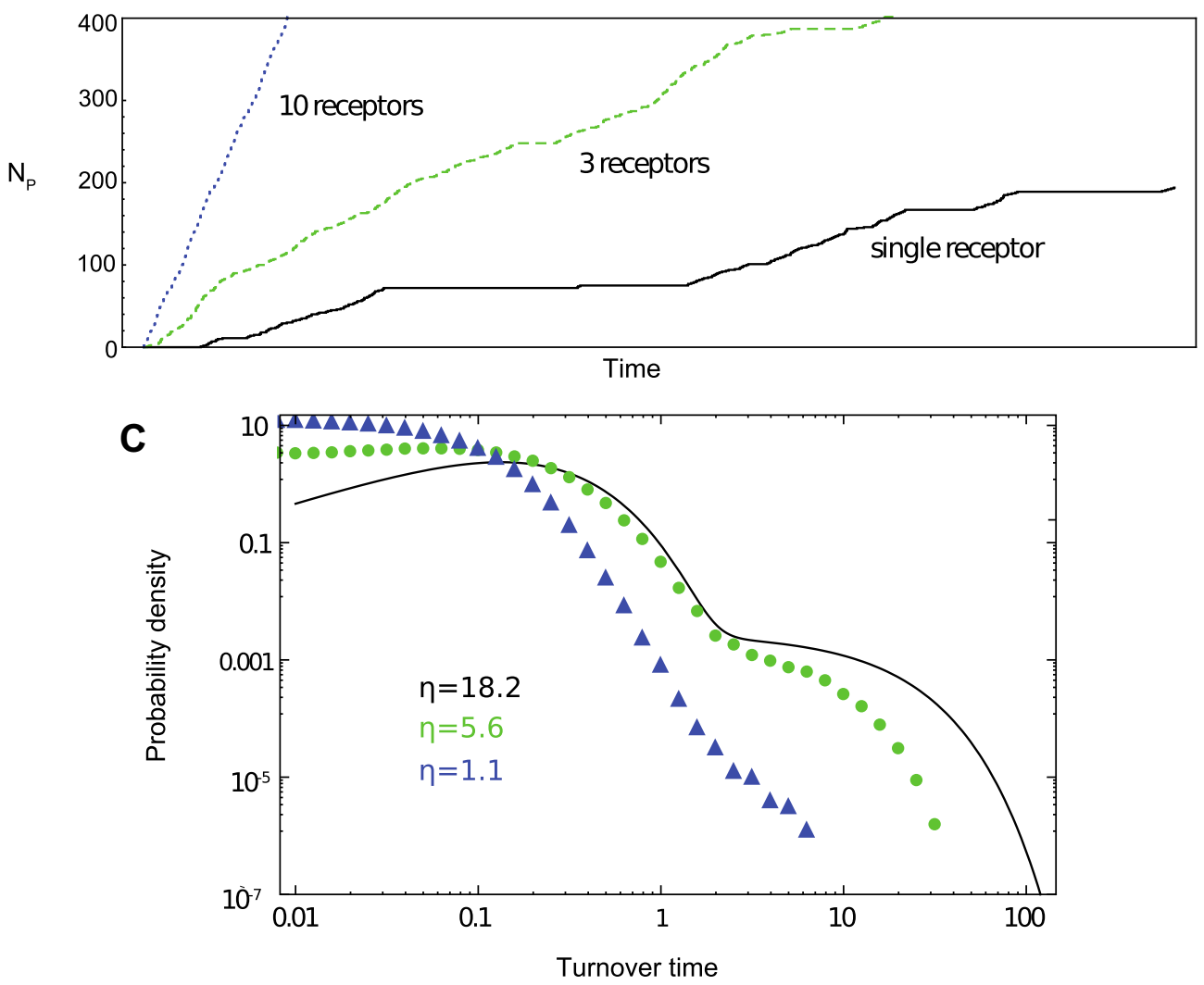

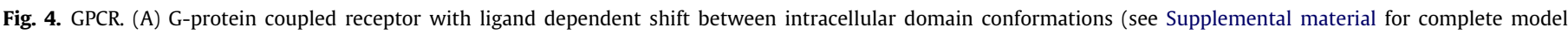

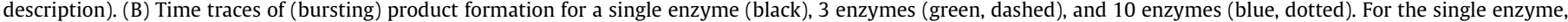

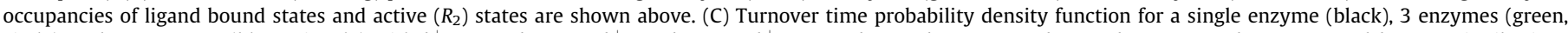

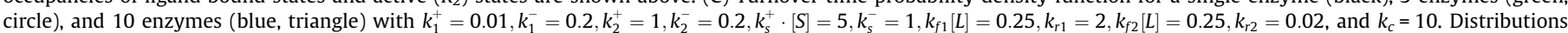

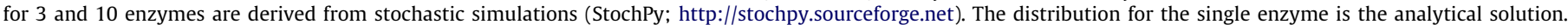
Noise in the turnover time distribution is indicated by $\eta$.

fractional error in concentration, $\delta c / c$, more if the averaging time is long in comparison to the time that it takes to replace the molecules in the receptor's detection volume by new ones (re-equilibration); because then several independent measurements are taken and averaged over. The time it takes to clear a volume of size $s^{3}$ by diffusion is proportional to $s^{2} / D$, with $D$ as the ligand diffusion coefficient. Exact coefficients again depend on the geometry of the volume; the efflux out of the volume is $D / s^{2} c=k c$ and the autocorrelation time then becomes $1 / k$. As a consequence, $X=T /\left(s^{2} / D\right)$ independent measurements of the molecule number are achievable in a time interval $T$. This reduces the error, $\delta n$, by a factor of $1 / X^{1 / 2}$ (the variance decreases by the factor $X^{-1}$ according to the 
central limit theorem). In total, the coefficient of variation - "error" - in the concentration measurement can, therefore, be expected to be equal to or higher than:

$\frac{\delta n}{n}=\frac{\delta c}{c}=\frac{\left(c s^{3}\right)^{1 / 2}}{\left(c s^{3}\right) X^{1 / 2}}=\frac{1}{\sqrt{T /\left(s^{2} / D\right) c s^{3}}}=\frac{1}{\sqrt{D s c T}}$

A similar result can also be derived from a slightly different perspective: The rate with which "new" molecules, i.e., molecules that have not bound to the receptor before, arrive at a single receptor is proportional to $4 D s c$. The exact expression depends on the geometry of the receptor (leading to differences between Eqs. (15) and (16)). If the receptor is occupied with probability $p$, the number of unique molecules bound to the receptor during a time interval $T$ therefore is proportional to $4 \operatorname{Dsc}(1-p) T$. Due to counting noise alone, the minimum fractional error in the determination of the receptor is:

$\frac{\delta c}{c}=\frac{1}{\sqrt{2(1-p)} \sqrt{D s c T}}$

The error could be higher than this noise floor, but as shown in [37] neither stirring of the medium nor swimming of the organism can significantly improve this bound. The two ways in which the fractional error can be minimized is by either taking longer time averages (increasing $T$ ) or by using multiple receptors.

Also in the case of multiple receptors only "new" molecules improve the fractional error on the cell's estimation of the concentration, "new" meaning here that a molecule did not bind to any of the cell's receptors before ("double counting"). The flux with which such molecules arrive can be calculated exactly for the case that receptors are distributed uniformly across the surface of a spheric cell and equals [37]:

$J=4 \pi a D c \frac{N s a}{N s+\pi a}$

Here, $N$ denotes the number of receptor molecules and $a$ the radius of the cell. If receptors are distributed randomly rather than uniformly, the flux decreases only by a few percent. Whereas a cluster of receptors receives a much lower flux of new molecules. The maximum flux of molecules would be achieved if the surface was completely covered with receptors. Interestingly, half of the maximum flux is already achieved with $N=\pi a / s$ receptors covering only about $1 / 1000$ of the cell's surface area. Also in the case of multiple receptors the fractional error in concentration is equal to $\frac{\delta c}{c}=(J(1-p) T / 2)^{-1 / 2}$.

Some of these ideas have been tested experimentally on a single-molecule level with the G-protein coupled cAMP receptor in Dictyostelium $[39,40]$. The ligand cAMP as well as different proteins in the signaling cascade, including the receptor and a few downstream messengers, were labeled fluorescently and monitored with total internal reflection fluorescence microscopy. In this way binding kinetics of the ligand as well as downstream signaling events could be followed on a single-molecule level. The experimental data confirmed the fundamental limits and showed that the chemotactic system in Dictyostelium operates remarkably close to these limits set by physics.

\section{Concluding remarks}

Enzymes are the workhorses of cells; essentially all cellular processes are catalyzed by enzymes. Classically, enzymes have been modeled with deterministic, macroscopic descriptions using ordinary differential equations leading to the well-known equilibrium-binding and steady-state models of enzyme kinetics [41]. Single-molecule measurement methods have lead to the study of the activity of single enzyme molecules $[13,14]$. These studies give new insights into the roles of the fluctuating, catalytic activity of enzymes due to the inherent stochasticity of enzyme-state transitions, fluctuation in enzyme conformations, and in the substrates of enzymes. Each of these aspects we have discussed in this work using small mathematical models to introduce several of the main concepts of this field to a wider audience.

The study of single-enzyme molecules indicates how molecular processes in cells that are reliant on single-enzyme activity generate "noise" that can propagate to the phenotype. On top of that noise is generated at the network level to give rise to significant levels of non-genetic phenotypic heterogeneity, which is playing a remarkably important role in the life of single cells [42].

\section{Acknowledgments}

T.M. is funded by the research program of BioSolar Cells, co-financed by the Dutch Ministry of Economic Affairs, Agriculture and Innovation. A.S. and F.B. acknowledge funding of NWO-VIDI Project 864.11.011.

\section{Appendix A. Supplementary data}

Supplementary data associated with this article can be found, in the online version, at http://dx.doi.org/10.1016/j.febslet.2013. 07.005.

\section{References}

[1] Michaelis, L. and Menten, M.L. (1913) Die Kinetik der Invertinwirkung. Biochem. Z. 49, 333-369.

[2] Briggs, G.E. and Haldane, J.B. (1925) A note on the kinetics of enzyme action. Biochem. J. 19 (2), 338-339.

[3] Cleland, W.W. (1963) The kinetics of enzyme-catalyzed reactions with two or more substrates or products. I. Nomenclature and rate equations. Biochim. Biophys. Acta 67, 104-137.

[4] Cleland, W.W. (1963) The kinetics of enzyme-catalyzed reactions with two or more substrates or products. II. Inhibition: nomenclature and theory. Biochim. Biophys. Acta 67, 173-187.

[5] Cleland, W.W. (1963) The kinetics of enzyme-catalyzed reactions with two or more substrates or products. III. Prediction of initial velocity and inhibition patterns by inspection. Biochim. Biophys. Acta 67, 188-196.

[6] Umbarger, H.E. (1956) Evidence for a negative-feedback mechanism in the biosynthesis of isoleucine. Science 123 (3202). 848-848.

[7] Pardee, A.B. and Yates, R.A. (1956) Control of pyrimidine biosynthesis in Escherichia coli by a feed-back mechanism. J. Biol. Chem. 221 (2), 757-770.

[8] Perutz, M.F. (1989) Mechanisms of cooperativity and allosteric regulation in proteins. Q. Rev. Biophys. 22 (2), 139-237.

[9] Changeux, J.P. (2012) Allostery and the Monod-Wyman-Changeux model after 50 years. Annu. Rev. Biophys. 41, 103-133.

[10] Monod, J., Wyman, J. and Changeux, J.P. (1965) On the nature of allosteric transitions: a plausible model. J. Mol. Biol. 12, 88-118.

[11] Koshland, D.E., Némethy, G. and Filmer, D. (1966) Comparison of experimental binding data and theoretical models in proteins containing subunits. Biochemistry 5 (1), 365-385.

[12] Hofmeyr, J.H. and Cornish-Bowden, A. (1997) The reversible Hill equation: how to incorporate cooperative enzymes into metabolic models. Comput. Appl. Biosci. 13 (4), 377-385.

[13] English, B.P., Min, W., van Oijen, A.M., Lee, K.T., Luo, G., Sun, H., Cherayil, B.J., Kou, S.C. and Xie, X.S. (2006) Ever-fluctuating single enzyme molecules: Michaelis-Menten equation revisited. Nat. Chem. Biol. 2 (2), 87-94.

[14] Claessen, V.I., Engelkamp, H., Christianen, P.C., Maan, J.C., Nolte, R.J., Blank, K. and Rowan, A.E. (2010) Single-biomolecule kinetics: the art of studying a single enzyme. Annu. Rev. Anal. Chem. (Palo Alto Calif.) 3, 319-340.

[15] Rotman, B. (1961) Measurement of activity of single molecules of beta-Dgalactosidase. Proc. Natl. Acad. Sci. U. S. A. 47, 1981-1991.

[16] McQuarrie, D. (1967) Stochastic approach to chemical kinetics. J. Appl. Probab. 4, 413.

[17] Qian, H. and Bishop, L.M. (2010) The chemical master equation approach to nonequilibrium steady-state of open biochemical systems: linear singlemolecule enzyme kinetics and nonlinear biochemical reaction networks. Int. J. Mol. Sci. 11 (9), 3472-3500.

[18] Berg, O.G. and von Hippel, P.H. (1985) Diffusion-controlled macromolecular interactions. Annu. Rev. Biophys. Biophys. Chem. 14, 131-160.

[19] Qian, H. (2008) Cooperativity and specificity in enzyme kinetics: a singlemolecule time-based perspective. Biophys. J. 95 (1), 10-17.

[20] Garai, A., Chowdhury, D., Chowdhury, D. and Ramakrishnan, T.V. (2009) Stochastic kinetics of ribosomes: single motor properties and collective behavior. Phys. Rev. E Stat. Nonlin. Soft Matter Phys. 80 (1 Pt 1), 011908. 
[21] Keller, D. and Bustamante, C. (2000) The mechanochemistry of molecular motors. Biophys. J. 78 (2), 541-556.

[22] Velonia, K., Flomenbom, O., Loos, D., Masuo, S., Cotlet, M., Engelborghs, Y., Hofkens, J., Rowan, A.E., Klafter, J., Nolte, R.J. and de Schryver, F.C. (2005) Single-enzyme kinetics of CALB-catalyzed hydrolysis. Angew. Chem. Int. Ed. Engl. 44 (4), 560-564.

[23] Lu, H.P., Xun, L. and Xie, X.S. (1998) Single-molecule enzymatic dynamics. Science 282 (5395), 1877-1882.

[24] Flomenbom, O., Velonia, K., Loos, D., Masuo, S., Cotlet, M., Engelborghs, Y., Hofkens, J., Rowan, A.E., Nolte, R.J., Van der Auweraer, M., de Schryver, F.C. and Klafter, J. (2005) Stretched exponential decay and correlations in the catalytic activity of fluctuating single lipase molecules. Proc. Natl. Acad. Sci. U. S. A. 102 (7), 2368-2372.

[25] Kuznetsova, S., Zauner, G., Aartsma, T.J., Engelkamp, H., Hatzakis, N., Rowan, A.E., Nolte, R.J., Christianen, P.C. and Canters, G.W. (2008) The enzyme mechanism of nitrite reductase studied at single-molecule level. Proc. Natl. Acad. Sci. U. S. A. 105 (9), 3250-3255.

[26] Saha, S., Sinha, A. and Dua, A. (2012) Single-molecule enzyme kinetics in the presence of inhibitors. J. Chem. Phys. 137 (4), 045102.

[27] Cao, J. (2011) Michaelis-Menten equation and detailed balance in enzymatic networks. J. Phys. Chem. B 115 (18), 5493-5498.

[28] Min, W., Gopich, I.V., English, B.P., Kou, S.C., Xie, X.S. and Szabo, A. (2006) When does the Michaelis-Menten equation hold for fluctuating enzymes? J. Phys. Chem. B 110 (41), 20093-20097.

[29] Xue, X., Liu, F. and Ou-Yang, Z.C. (2006) Single molecule Michaelis-Menten equation beyond quasistatic disorder. Phys. Rev. E Stat. Nonlin. Soft Matter Phys. 74 (3 Pt 1), 030902.

[30] Kou, S.C., Cherayil, B.J., Min, W., English, B.P. and Xie, X.S. (2005) Singlemolecule Michaelis-Menten equations. J. Phys. Chem. B 109 (41), 1906819081.
[31] Kolomeisky, A.B. (2011) Michaelis-Menten relations for complex enzymatic networks. J. Chem. Phys. 134 (15), 155101.

[32] Svoboda, K., Mitra, P.P. and Block, S.M. (1994) Fluctuation analysis of motor protein movement and single enzyme kinetics. Proc. Natl. Acad. Sci. U. S. A. 91 (25), 11782-11786.

[33] Du, C. and Kou, S.C. (2012) Correlation analysis of enzymatic reaction of a single protein molecule. Ann. Appl. Stat. 6 (3), 950-976.

[34] Dobrzynski, M. and Bruggeman, F.J. (2009) Elongation dynamics shape bursty transcription and translation. Proc. Natl. Acad. Sci. U. S. A. 106 (8), 2583-2588.

[35] Schwabe, A., Rybakova, K.N. and Bruggeman, F.J. (2012) Transcription stochasticity of complex gene regulation models. Biophys. J. 103 (6), 1152 1161.

[36] Cinlar, E. (1972) in: Stochastic Point Processes (Lewis, P., Ed.), pp. 546-606, Wiley Interscience.

[37] Berg, H.C. and Purcell, E.M. (1977) Physics of chemoreception. Biophys. J. 20 (2), 193-219.

[38] Bialek, W. and Setayeshgar, S. (2005) Physical limits to biochemical signaling. Proc. Natl. Acad. Sci. U. S. A. 102 (29), 10040-10045.

[39] Ueda, M., Sako, Y., Tanaka, T., Devreotes, P. and Yanagida, T. (2001) Singlemolecule analysis of chemotactic signaling in Dictyostelium cells. Science 294 (5543), 864-867.

[40] Miyanaga, Y. and Ueda, M. (2011) Single-molecule kinetic analysis of stochastic signal transduction mediated by G-protein coupled chemoattractant receptors in: Cell Signaling Reactions (Sako, Y. and Ueda, M., Eds.), pp. 33-57, Springer, The Netherlands.

[41] Cornish-Bowden, A. (1995) Fundamentals of Enzyme Kinetics, Portland Press.

[42] Schwabe, A., Dobrzyński, M., Rybakova, K., Verschure, P. and Bruggeman, F.J. (2011) Origins of stochastic intracellular processes and consequences for cellto-cell variability and cellular survival strategies. Methods Enzymol. 500, 597625. 